\title{
Skin Lesions Classification with Optical Spectroscopy
}

\author{
Asad Safi ${ }^{1}$, Victor Castaneda ${ }^{1}$, Tobias $\operatorname{Lasser}^{1}$ and Nassir Navab ${ }^{1}$ \\ ${ }^{1}$ Computer Aided Medical Procedures (CAMP), \\ Technische Universität München, Germany \\ \{safi, castaned, lasser\}@in.tum.de, navab@cs.tum.edu
}

\begin{abstract}
Diagnosis of benign and malign skin lesions is currently mostly relying on visual assessment and frequent biopsies performed by dermatologists. As the timely and correct diagnosis of these skin lesions is one of the most important factors in the therapeutic outcome, leveraging new technologies to assist the dermatologist seems natural. Complicating matters is a blood cancer called Cutaneous T-Cell Lymphoma, which also exhibits symptoms as skin lesions. We propose a framework using optical spectroscopy and a multi-spectral classification scheme using support vector machines to assist dermatologists in diagnosis of normal, benign and malign skin lesions. As a first step we show successful classification (94.9\%) of skin lesions from regular skin in 48 patients based on 436 measurements. This forms the basis for future automated classification of different skin lesions in diseased patients.
\end{abstract}

Keywords: Skin cancer, Optical spectroscopy, Classification.

\section{Introduction}

Skin cancer is one of the most common cancer types in humans and its incidence is on the rise, especially in countries where the ozone layer is thinning. The correct and timely diagnosis of suspicious skin lesions is one of the most important factors in the therapeutical outcome.

At present most dermatologists rely on their experience of visual assessment to distinguish benign and malign skin lesions [1] like pigmented nevi, seborrhoeic keratosis or basal cell carcinoma and malignant melanoma, as well as requiring biopsies of the affected skin.

To complicate matters, Cutaneous T-Cell Lymphoma (CTCL) is a blood cancer type with symptoms that are exhibited as skin lesions as well. Again a timely diagnosis and staging is very crucial for a successful treatment [2].

New technologies to assist in identifying and diagnosing skin lesion and to minimize invasive biopsies have been developed, like hand-held magnification devices and computer-aided image analysis. Colourd image processing methods were introduced for melanoma [3] which focused on non-constant visual information of skin lesions. Neural network diagnosis of skin lesion has previously been applied by classifying extracted features from digitized dermoscopy images of lesions [4][5]. The 
extracted features are based on geometry, colors, and texture of the lesions, involving complex image processing techniques. Recently with Raman spectroscopy the molecular structure of skin lesions are exploited [6], but due to harmful effect of laser beam on sensitive skin surface, is least in practice for dermatologist. Optical spectroscopy is another technology that is being established to aid in skin lesion diagnosis [7], as the multi-spectral nature of this imaging method allows to detect and classify multiple physiological changes like those associated with increased vasculature, cellular structure, oxygen consumption or edema in tumors [8].

We propose a computer-aided system using optical spectroscopy that keeps track of the progression of skin lesions and assists in quantification and classification of skin diseases in order to assist dermatologists in the decision making process. In this paper we present a framework for acquiring spectroscopic data of skin lesions and classifying them using support vector machines (SVM). We report on the classification results obtained from optical spectroscopy using various skin lesions of 48 patients.

In the following section we describe the materials and methods used in this study and the last section explains experiments and their results.

\section{Materials and Methods}

Here we describe the employed algorithms and the instrument used for data acquisition.

\subsection{System setup}

The system contains a hand-held reflectance spectroscopy probe (StellarNet Inc., Oldsmar, FL, USA), consisting of $6 \times 200 \mu \mathrm{m}$ illumination fibers arrayed around one $600 \mu \mathrm{m}$ acquisition fiber. The probe has an infrared optical tracking target attached in order to be able to determine its position and orientation in real-time, see fig. 1(a). The tracking system consists of four ARTtrack2 infrared cameras (A.R.T. GmbH, Weilheim, Germany) positioned to track within a volume of $2 \times 2 \times 2\left[\mathrm{~m}^{3}\right]$.

A 178-1132 [nm], 2048 [pix], 12bit CCD spectrometer (StellarNet Inc., Oldsmar, FL, USA) is connected to the acquisition fiber, and a 12 [W] tungsten lamp connected to the illumination fibers as a light source. The spectrometer is controlled by a data processing unit to acquire spectra synchronously with the tracking information of the probe. The data-processing unit is used to run the application that handles the incoming data (spectral and spatial) and the classification. An overview of the entire setup is displayed in fig. 1 (b). 


\subsection{Data Processing}

The spectral data is acquired as a 2048 vector of the floating points values $x_{i} \in R^{2048}, i=1, \ldots, n$ where $n$ denotes the number of measurements. Each $x_{i}$ represents the discretized reflective spectrum from 178 [nm] to 1132 [nm] (due to limitation of hardware )of the $i$ th measurement and is stored normalized as

$$
\hat{x}_{i}=\frac{x_{i}}{\left\|x_{i}\right\|_{2}} \quad \text { where } i=1 \ldots \mathrm{n}
$$

To reduce the dimensions of the input data, principal components analysis (PCA) is applied. The resulting spectrum of eigenvalues $\left(e_{j}^{i}\right)_{j=1, \ldots, 2048}$ is sorted descending by magnitude. Since the highest eigenvalues represent the most relevant components, a cut-off value $C_{P C A}$ is chosen, such that the final input data $\mathrm{y}_{\mathrm{i}}$ for the classification algorithm from measurement $x_{i}(i=1, \ldots, \mathrm{n})$ is

$$
y_{i}=\left(e_{j}^{i}\right)_{j=1, \ldots, C_{P C A}} \text {. }
$$

The cut-off value $C_{P C A}$ is chosen empirically from the data. Fig. 2 is showing a representative example of $\left(e_{j}^{i}\right)_{j=1, \ldots, 2048}$ from which $C_{P C A}$ was selected as one of $\{2,3,4,5\}$.
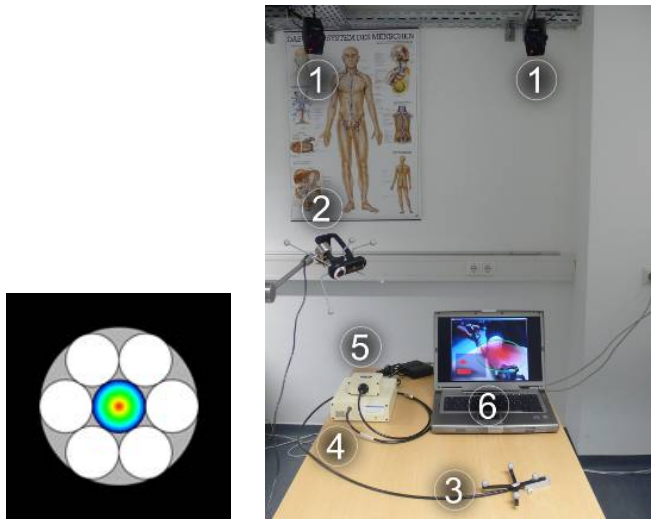

Fig. 1. (a) Schematic of the fiber arrangement in the spectroscopy probe: $6 \times 200 \mu \mathrm{m}$ illumination fibers arrayed around one $600 \mu \mathrm{m}$ acquisition fiber. (b) System setup: (1) tracking cameras, (2) regular camera (for augmented reality visualization), (3) tracked probe, (4) spectrometer, (5) light source, and (6) data-processing unit. 


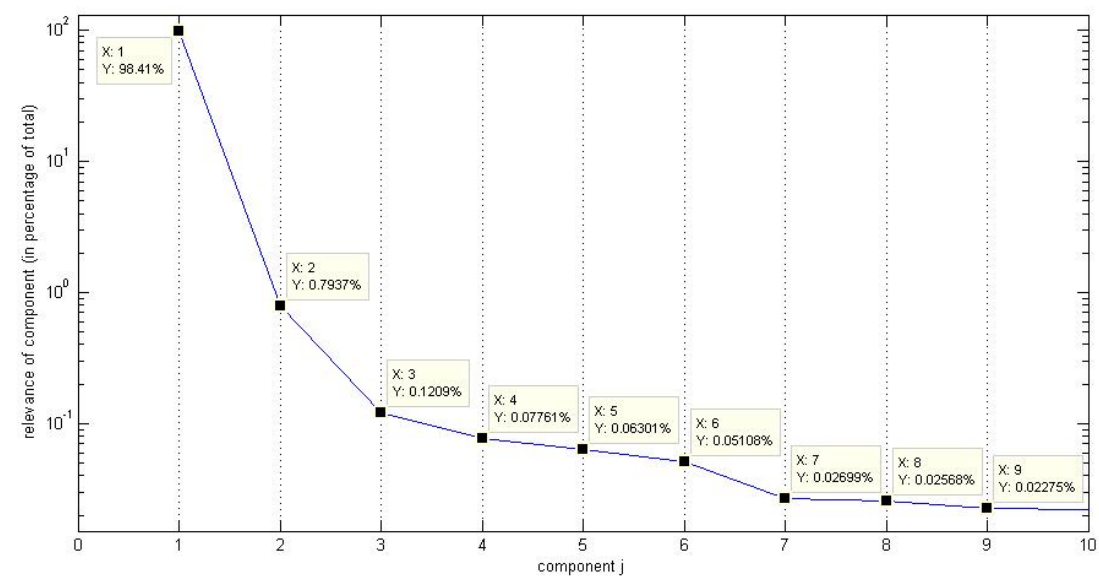

Fig. 2. Representative example of the first part of the sorted PCA eigenvalue spectrum $\left(e_{j}^{i}\right)$, the $\mathrm{y}$-axis shows the values of the component as a percentage of the total in $\log$ scale.

\subsection{Classification}

Classification is performed by a support vector machine (SVM), [9]. SVM was selected as the method of choice as it allows to linearly classify data in a highdimensional feature space that is non-linearly related to the input space via the use of specific kernel functions, such as polynomial functions or radial basis functions (RBF). This way we can build complex enough models for skin lesion classification while still being able to compute directly in the input space.

The SVM classifier needs to be trained first before using it, thus we partition our (already reduced) input data $\left(y_{i}\right), i=1, \ldots, n$ into two partitions, $T \subset\{1, \ldots, n\}$ the training set and $V \subset\{1, \ldots, n\}$ the testing (or validation) set with $T \cup V=\{1, \ldots, n\}$ and $T \cap V=\{\}$. The training data set $T$ is labeled manually into two classes with the ground truth, $l\left(y_{i}\right)= \pm 1$. Once the classifier is trained, a simple evaluation of the decision function $d\left(y_{i}\right)= \pm 1$ will yield the classification of any data $y_{i}$.

In detail, SVM is trying to separate the data $\phi\left(y_{i}\right)$ mapped by the selected kernel function $\phi$ by a hyperplane $w^{T} \phi\left(y_{i}\right)+b=0$ with $w$ the normal vector and $b$ the translation. The decision function then is $d\left(y_{i}\right)=\operatorname{sgn}\left(w^{T} \phi\left(y_{i}\right)+b\right)$. Maximizing the margin and introducing slack variables $\xi=\left(\xi_{i}\right)$ for non-separable data, we receive the primal optimization problem: 


$$
\begin{gathered}
\min _{w, b, \xi}=\frac{1}{2} w^{T} w+C \sum_{i \in T} \xi_{i} \\
\text { with constrains } l\left(y_{i}\right)\left(w^{T} \phi\left(y_{i}\right)+b\right) \geq 1-\xi_{i} \\
\xi_{i} \geq 0 \text { for } i \in T,
\end{gathered}
$$

where $C$ is a user-determined penalty parameter. Switching to the dual optimization problem allows for easier computation,

$$
\begin{aligned}
\min _{\alpha}=\frac{1}{2} \alpha^{T} Q \alpha-e^{T} \alpha \\
\text { with constrains } 0 \leq \alpha_{i} \leq C \text { for } i \in T \\
\sum_{i \in T} y_{i} \alpha_{i}=0,
\end{aligned}
$$

where $\alpha=\left(\alpha_{i}\right)$ are the so-called support vectors, $e=[1, \ldots, 1]^{T}$ and $Q$ is the positive semidefinite matrix formed by $Q_{j k}=l\left(y_{j}\right) l\left(y_{k}\right) K\left(y_{j}, y_{k}\right)$, and $K\left(y_{j}, y_{k}\right)=\phi\left(y_{j}\right)^{T} \phi\left(y_{k}\right)$ is the kernel function built from $\phi$. Once this optimization problem is solved, we determine the hyperplane parameters $w$ and $b, w$ directly as $w=\sum_{i \in T} \alpha_{i} l\left(y_{i}\right) \phi\left(y_{i}\right)$ and $b$ via one of the Karush-Kuhn-Tucker conditions as $b=-l\left(y_{j}\right) y_{i}^{T} w$, for those $i$ with $0<\alpha_{i}<C$. Thus the decision function of the trained SVM classifier ends up as

$$
d\left(y_{i}\right)=\operatorname{sgn}\left(w^{T} \phi\left(y_{j}\right)+b\right)=\operatorname{sgn}\left(\sum_{j \in T} \alpha_{j} l\left(y_{j}\right) K\left(y_{j}, y_{j}\right)+b\right) .
$$

\section{Experiments and Results}

\subsection{Experiments}

We collected 436 spectroscopic data points $\left(x_{i}\right), i=1, \ldots, 436$ from the skin of 48 patients, 326 measurements were of skin lesions, 110 measurements were of normal skin. Exemplary picture of the lesion imaged is shown in fig. 4.

All data was manually labeled into the two classes normal skin $l\left(x_{i}\right)=1$ and lesion $l\left(x_{i}\right)=-1$. The 436 data points were randomly separated into a training data set $T$ and a testing (validation) data set $V$ with $|T|=305$ and $|V|=131$, however retaining the balance of both sets containing $50 \%$ each of the two classes. A color-coded representation of the normalized skin spectra $\hat{x}_{i}, i \in T$, of the training data set $T$ is shown in fig. 5. 
Before classification, PCA was applied to the $\hat{x}_{i}$ for dimension reduction to yield our classification input $y_{i}$. The eigenvalue cut-off $C_{P C A}$ was empirically chosen as one of $C_{P C A} \in\{2,3,4,5\}$.

The SVM classifier (we used C-SVM from LibSVM, [10]) was then trained using the training data set $T$. As there are multiple parameters to be selected, like for example the penalty parameter $C$, we performed a cross-validation of 3 folds via parallel grid search. The average accuracy on the prediction of the validation fold is the cross validation accuracy.

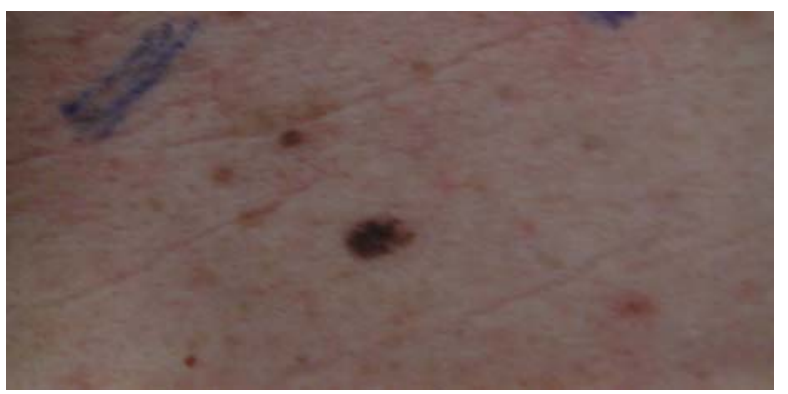

Fig. 4. Skin moles
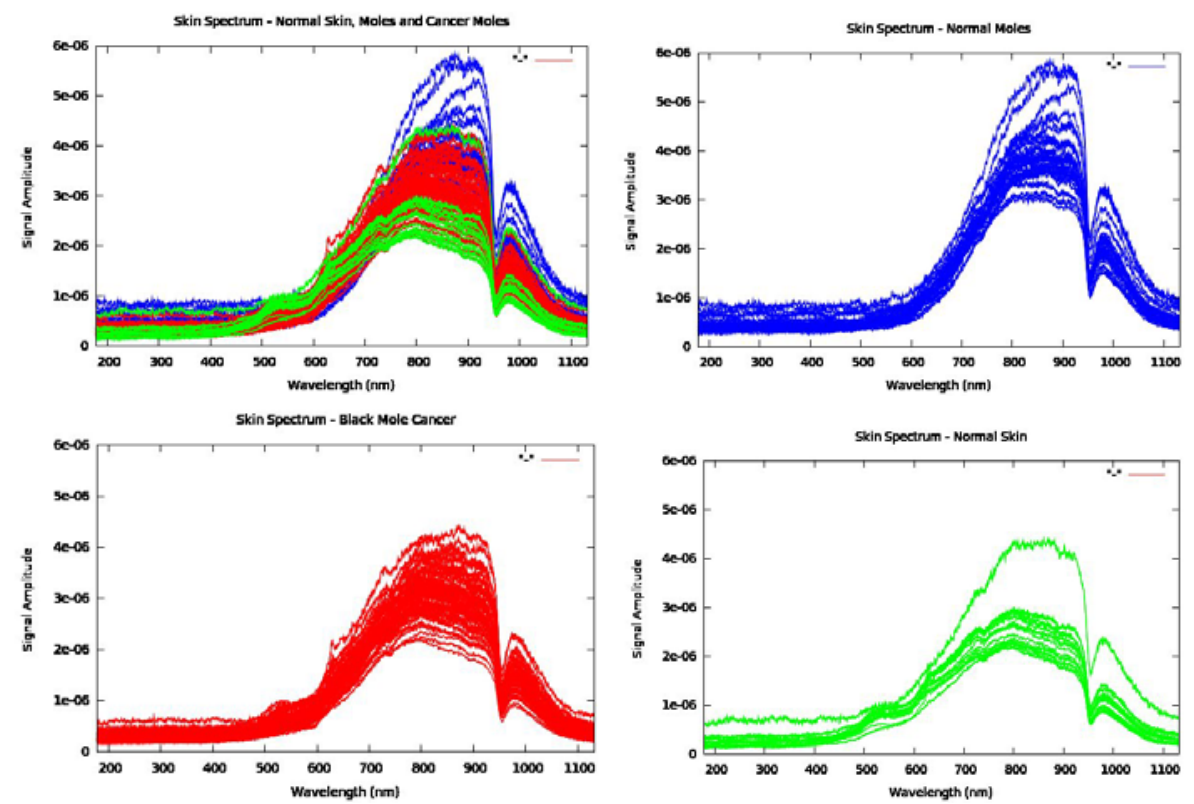

Fig. 5. Graph of all normalized spectra $\hat{x}_{i}$ from the training data set $T$, color-coded as blue for skin moles, red mole cancer and green for normal skin. 


\subsection{Results}

The cross-validation of the training data set $T$ determined, among others, the parameters $C=-5$ and $\gamma=-7$. For the further parameters $C_{P C A}$ and the choice of the kernel (linear, polynomial, radial basis function (RBF) or sigmoid) we performed cross validation of the training data set $T$, the results are shown in Table 1 . The best results were received consistently by using the RBF kernel, while for $C_{P C A}$ the value of 5 turned out to be the best choice with an accuracy of $97 \pm 8.3$.

Table 1. Results of the cross-validation using the training dataset $T$.

\begin{tabular}{|l|l|l|l|l|}
\hline \multicolumn{5}{|c|}{ C-SVC Training } \\
\hline & Linear Kernel & Poly Kernel & RBF Kernel & Sigmoid Kernel \\
\hline$C_{P C A}=2$ & $95 \pm 9.2$ & $96 \pm 8.3$ & $95 \pm 7.5$ & $95 \pm 10.1$ \\
\hline$C_{P C A}=3$ & $95 \pm 8.3$ & $96 \pm 6.7$ & $97 \pm 9.5$ & $96 \pm 10.5$ \\
\hline$C_{P C A}=4$ & $95 \pm 11.5$ & $97 \pm 7.2$ & $97 \pm 8.7$ & $96 \pm 8.6$ \\
\hline$C_{P C A}=5$ & $96 \pm 9.2$ & $97 \pm 10.5$ & $97 \pm 8.3$ & $97 \pm 7.7$ \\
\hline
\end{tabular}

With the training of the classifier completed, we studied the accuracy of the testing (validation) data set $V$. We compared the manual ground truth labeling $l(y i)$ for data point $y_{i}$ with the computed decision function $d\left(y_{i}\right)$ to compute the accuracy as follows

$$
\text { Accuracy }=\frac{\# \text { of correctly predicted data }}{\# \text { total data }} \times 100 \%=\frac{\left|\left\{i \mid l\left(y_{i}\right) d\left(y_{i}\right)>0\right\}\right|}{|V|} \times 100 \%
$$

Table 2. Classification accuracy results using the testing dataset $V$.

\begin{tabular}{|l|l|l|l|l|}
\hline \multicolumn{2}{|c|}{ Testing } & \\
\hline & Linear Kernel & Poly Kernel & RBF Kernel & Sigmoid Kernel \\
\hline$C_{P C A}=2$ & $86.8 \%$ & $90.3 \%$ & $89.9 \%$ & $88.8 \%$ \\
\hline$C_{P C A}=3$ & $89.3 \%$ & $92.5 \%$ & $91.8 \%$ & $90.3 \%$ \\
\hline$C_{P C A}=4$ & $91.9 \%$ & $92.9 \%$ & $94.9 \%$ & $94.1 \%$ \\
\hline$C_{P C A}=5$ & $92.1 \%$ & $93.6 \%$ & $94.9 \%$ & $94.6 \%$ \\
\hline
\end{tabular}

The results are shown in Table 2. We achieve the same accuracy of $94.9 \%$ for the kernels RBF the $C_{P C A}$ values of 4 and 5. This corresponds to fig. 2, where it is clear that between $C_{P C A} 4$ and 5 there is only very little difference. In total we received the best results using the RBF kernel and $C_{P C A}=5$. 


\section{Conclusion}

We present a portable, affordable setup for optical spectroscopy and SVM-based classification of skin lesions. Our experiments on patient's data served as a base to choose and tune the various parameters in the classification chain. The results of $94.9 \%$ accuracy in distinguishing normal skin from any type of skin lesion are comparable to those of a well-trained dermatologist using visual inspection [11].

Future work includes considering some factors which have not been addressed yet, such as the influence of external light and obstruction by hair (all measurements avoided hair) and comparison with other techniques such as neural networks and manifold learning. The next step then is to established complete framework for clinical evaluation in dermatology department and its determined parameters to classify different skin lesions of diseased patients. These results promise that computer-assisted multi-spectral imaging and classification is the path into the future for dermatological screening.

\section{References}

1. B. Lindelöf and M. Hedblad.: Accuracy in the clinical diagnosis and pattern of malignant melanoma at a dermatological clinic. J. Dermatol., vol. 21, no. 7, pp. 461-464, (1994)

2. Klemke, C.D., Goerdt, S., Schrama, D., Becker, J.C.: New insights into the molecular biology and targeted therapy of cutaneous t-cell lymphomas. Journal der Deutschen Dermatologischen Gesellschaft 4(5) 395-406 (2006)

3. O. Colot, R. Devinoy, A. Sombo and D. de Brucq.: A color image processing method for melanoma detection Medical Image Computing and Computer-Assisted Interventation MICCAI, 978-3-540-65136-9, (1998)

4. M. Hintz-Madsen, L. Hansen, J. Larsen, and K. Drzewiecki.: A probabilistic neural network framework for detection of malignant melanoma, in artificial neural networks in cancer diagnosis, prognosis and patient management. R. Naguib and G. Sherbet, Eds. Boca Raton, FL: CRC, pp. 141-183. (2001)

5. P. Rubegni, M. Burroni, G. Cevenini, R. Perotti, G. Dell'Eva, P. Barbini, M. Fimiani, and L. Andreassi.: Digital dermoscopy analysis and artificial neural network for the differentiation of clinically atypical pigmented skin lesions: A retrospective study.: J. Investigat. Dermatol., vol. 119, pp. 471-474, (2002)

6. S. Sigurdsson, P. Philipsen, L. Hansen, J. Larsen, M. Gniadecka, and H. Wulf.: Detection of Skin Cancer by Classification of Raman Spectra. IEEE transactions on biomedical engineering, No 10, vol. 51, (2004)

7. McIntosh, L.M., Summers, R., Jackson, M., Mantsch, H.H., Mansfield, J.R., Howlett, M., Crowson, A.N., Toole, J.W.: Towards non-invasive screening of skin lesions by nearinfrared spectroscopy. J Invest Dermatol 116(1) 175-181, (2001)

8. A. Kim, U. Kasthuri, B.C. Wilson, A. White, A. L. Martel.: Preliminary Clinical Results for the In Vivo Detection of Breast Cancer Using Interstitial Diffuse Optical Spectroscopy. MICCAI Workshop on Biophotonics Imaging for Diagnostics and Treatment, ISSN 16012321, 75 (2006)

9. Nello Cristianini and John Shawe-Taylor.: An Introduction to Support Vector Machines and Other Kernel-based Learning Methods. Cambridge University Press ISBN:0521780195, (2000) 
10. Chih-Chung Chang and Chih-Jen Lin, LIBSVM: A library for support vector machines, (2001). Open source available at http://www.csie.ntu.edu.tw/ cjlin/libsvm

11. Morton C A and Mackie R M.: Clinical accuracy of the diagnosis of cutaneous malignant melanoma Br. J. Dermatol. 138 283-7 (1998) 